\title{
Vespas Sociais (Vespidae: Polistinae) em uma Área de Floresta Ombrófila Densa Amazônica no Estado do Maranhão, Brasil
}

\author{
Alexandre Somavilla1 ${ }^{\bowtie}$, Dayse Willkenia Almeida Marques ${ }^{1}$, Ernesto Augusto Silva Barbosa ${ }^{1}$, \\ Juarez da Silva Pinto Junior² \& Marcio Luiz de Oliveira ${ }^{1}$
}

\begin{abstract}
1. Instituto de Pesquisas da Amazônia, Programa de Pós-Graduação em Entomologia, e-mail: alexandre.s@hotmail.com (Autor para correspondência ${ }^{\varpi}$ ), daysewillkenia@hotmail.com, barbosa.insects@gmail.com, mlolivei@inpa.gov.br. 2. Universidade Estadual do Maranhão, e-mail: j.pintojunior@hotmail.com.
\end{abstract}

\section{EntomoBrasilis 7 (3): 183-187 (2014)}

Resumo. As vespas sociais constituem um grupo com elevada riqueza de espécies e muito comum em áreas amazônicas. Apesar disto, nenhum trabalho foi realizado na Amazônia Maranhense e apenas 58 espécies de vespas sociais são registradas para o estado do Maranhão. O presente trabalho apresenta dados faunísticos referentes às vespas sociais da Reserva Biológica do Gurupi, caracterizada por ser uma floresta ombrófila latifoliada amazônica localizada no noroeste do Maranhão. As coletas e instalação de armadilhas (Malaise do tipo Gressitt \& Gressitt, armadilha suspensa, armadilha luminosa, armadilha atrativa com isca de fruta) ocorreram entre janeiro de 2010 e maio de 2011, totalizando 63 dias amostrados. Para a Reserva Biológica do Gurupi foram obtidos 384 espécimes de vespas sociais pertencentes a Epiponini e Polistini, alocados em 12 gêneros e 38 espécies. Polybia destacou-se por apresentar o maior número de espécies (14), seguido de Agelaia (seis espécies) e Apoica (quatro espécies). Quatorze espécies são registradas pela primeira vez para o estado do Maranhão, aumentando, desta forma, para 72 espécies com registros confirmados para o estado.

Palavras-chave: Amazônia Maranhense; Diversidade; Polybia; Reserva Biológica do Gurupi.

\section{Social Wasps (Vespidae: Polistinae) in a Dense Ombrophilous Amazon Forest in the Maranhão State, Brazil}

Abstract. Social wasps are a high species richness group and very common in Amazonian areas. Despite this, no study have been made in the amazonian forest on the state of Maranhão and only 58 species of social wasps are registered for this state. This paper presents data about the social wasps fauna of Gurupi Biological Reserve, characterized as an ombrophylous broadleaf forest located in the northwest Amazon of Maranhão. The collect and traps installation (Malaise trap, suspended trap, light traps and fruit attractive trap) occurred between January 2010 and May 2011, with a total of 63 sample days. We collected 384 specimens in the Biological Reserve of Gurupi, were obtained social wasps belonging to Epiponini and Polistini, allocated to 12 genera and 38 species. Polybia have the largest number of species (14), followed by Agelaia (six species) and Apoica (four species). Fourteen species are first records for the state of Maranhão, increasing, thus, for 72 species with confirmed records occurrence to Maranhão.

Keywords: Amazon of Maranhão; Biological Reserve of Gurupi; Diversity; Polybia.

( representantes de Vespidae são popularmente conhecidos como vespas, marimbondos ou cabas. Apesar da maioria das espécies ocorrerem em ambientes naturais, algumas costumam ocorrer em áreas antropizadas, atuando de forma decisiva no equilíbrio trófico das comunidades de artrópodes. A ambiguidade alimentar das vespas as coloca em situação privilegiada para estudos sobre teias alimentares, como herbívoras, na coleta de néctar e pólen ou predadoras, na captura de larvas e insetos menores, tornando-se importantes controladores biológicos (Raposo Filho \& Rodrigues 1983a, 1983b; RESENDE et al. 2001).

Além disso, os vespídeos podem exercer importante função no transporte do pólen, fazendo parte da comunidade de polinizadores de algumas espécies vegetais (HeRmEs \& KöHLER 2006; SÜHs et al. 2009; Somavilla \& KöHLER 2012). Algumas espécies de vespídeos são sensíveis a mudanças ambientais, podendo ser utilizadas como bioindicadores ambientais (LASALLE 1993).

A família Vespidae conta com mais de 4.600 espécies descritas para o mundo, sendo especialmente comuns na região Tropical. Composta por seis subfamílias monofiléticas; três têm comportamento solitário ou, eventualmente, subsocial
(Eumeninae, Euparagiinae e Masarinae) e outras três têm comportamento social (Polistinae, Stenogastrinae e Vespinae). No Brasil, ocorrem apenas Eumeninae, Masarinae e Polistinae (CArpenter \& Marques 2001; Carpenter 2004).

Aproximadamente 940 espécies de Polistinae são válidas e a fauna brasileira de vespas sociais é a mais rica do mundo com 319 espécies. As espécies brasileiras desta subfamília pertencem a três tribos: Mischocyttarini (Mischocyttarus e 117 espécies), Polistini (Polistes e 38 espécies) e Epiponini (19 gêneros e 164 espécies) (CARPENTER \& MARQues 2001; CARPENTER 2004).

Apesar de ser um grupo com elevada riqueza de espécies e muito comum em áreas amazônicas, o conhecimento sobre a diversidade e biologia desse táxon na região ainda é bastante precário. $\mathrm{Na}$ Amazônia Brasileira, 20 gêneros e aproximadamente 200 espécies foram registrados, representando cerca de $70 \%$ da fauna brasileira de vespas sociais (SilveIra 2002), mas os estudos ainda são bastante localizados. Destacam-se os inventários realizados na Ilha de Maracá em Roraima (RAw 1998), em Caxiuanã no Pará

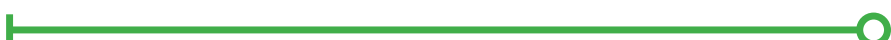

Agência(s) de Financiamento: Programa de Pesquisa em Biodiversidade (PPBio) e ao CNPq/MCTI - Amazônia Oriental (Processo 558287/2009-3) 
(Silveira 2002; Silva \& Silveira 2009), na Serra do Divisor no Acre (Morato et al. 2008), na região dos Lagos no Amapá (Silveira et al. 2008), em Mamirauá e Alvarães no Amazonas (Silveira et al. 2008) e Reserva Ducke no Amazonas (Somavilla 2012).

Para o estado do Maranhão, RichARDS (1978) reporta a ocorrência de 48 espécies de vespas sociais em diversas áreas e, recentemente, Silva et al. (2012) relatam a ocorrência de 31 espécies para uma área de Cerrado também neste estado, porém nenhum estudo foi realizado no domínio Amazônico. De acordo com estes dois estudos, 58 espécies possuem ocorrência confirmada para o Maranhão. Desta forma, o objetivo deste trabalho foi informar, pela primeira vez, a fauna de vespas sociais (Vespidae: Polistinae) de uma área de floresta Amazônica no Estado do Maranhão e discutir quais os métodos de coleta foram mais viáveis nesta localidade.

\section{MATERIAL E MÉTODOS}

Área de Estudo. A Reserva Biológica do Gurupi (REBIO do Gurupi) foi criada em 1988 e possui uma área de 341.650 hectares e é um dos últimos remanescentes de Floresta Amazônica situados no estado do Maranhão. Localizada entre os municípios de Centro Novo do Maranhão, Bom Jardim e São João do Carú (Figura 1) é considerada uma área prioritária para a conservação por fazer parte do Centro de Endemismo Belém, localizado na zona fisiográfica do leste do Pará e oeste do Maranhão (IBAMA 1999).

Predomina o clima tropical quente e úmido (As), típico da região amazônica. A temperatura média anual varia entre $25^{\circ} \mathrm{C}$ e $27^{\circ} \mathrm{C}$; umidade relativa do ar entre $76 \%$ e $79 \%$ e os totais pluviométricos variam entre 1.200 e $2.000 \mathrm{~mm}$. A região apresenta dois períodos bem distintos: junho a novembro, estação seca e dezembro a maio, estação chuvosa; o trimestre mais seco vai de julho a setembro e o mais chuvoso de fevereiro a abril. A Amazônia Maranhense é caracterizada por fragmentos de florestas ombrófilas, latifoliadas, distribuídas entre áreas úmidas e terra firme (IBAMA 1999).

Coleta. A instalação de armadilhas ocorreu entre janeiro de 2010 a maio de 2011 (numa frequência a cada dois meses), somando nove expedições com duração média de sete dias consecutivos, totalizando 63 dias amostrados.

Para a captura dos espécimes foram utilizados diferentes métodos indiretos como: armadilhas de Malaise do tipo GREssiTT \& GressitT (1962) (para a coleta de insetos que tendem a se deslocar pelo sub-bosque da floresta), armadilhas suspensa (um tipo de armadilha Malaise modificada, possuindo um septo preto com o intuito de coletar insetos que tendem a forragear nos estratos superiores da floresta e no dossel) (RAFAEL \& Gorayeb 1982); armadilha luminosa (que consistiu em um lençol branco de 300 x $200 \mathrm{~cm}$, com lâmpada mista de vapor de mercúrio de $250 \mathrm{~W}$, montados a cerca de $50 \mathrm{~cm}$ do solo, para coleta de insetos atraídos pela luz funcionando das $18 \mathrm{~h}$ até $06 \mathrm{~h}$ do dia seguinte) e armadilhas atrativas do tipo McPhail instaladas a 1,80 $\mathrm{m}$ do solo e $100 \mathrm{~m}$ de distância entre si, contendo $500 \mathrm{~mL}$ de isca atrativa de suco de laranja (60\% de polpa, 30\% de água e mais 10\% de açúcar) fermentado durante três dias (armadilha é utilizada para coletar insetos com dieta glicídica e que tendem a se alimentar de frutas). Foram instaladas, em cada uma das nove expedições, um conjunto de armadilhas que consistiam em uma armadilha Malaise, uma armadilha suspensa, trinta armadilhas atrativas, além da utilização de uma armadilha luminosa.

Complementar a estes métodos indiretos, no momento de instalação das armadilhas na área de estudo, algumas colônias de vespas sociais foram coletadas na totalidade ou coletados alguns indivíduos (em caso de colônias grandes com muitos indivíduos) o que resultou no registro das espécies, sendo que o encontro de uma colônia correspondia a um registro na contagem geral dos indivíduos.

Identificação. Todos os espécimes coletados foram incorporados ao acervo da Coleção Zoológica do Maranhão (CZMA), situada na Universidade Estadual do Maranhão, campus Caxias (UEMA), e na Coleção Zoológica de Invertebrados do Instituto Nacional de Pesquisas da Amazônia (INPA).

As identificações dos espécimes foram feitas com base em chaves propostas por RichardS (1978), CARPENTER \& MARQues (2001), CARPENTER (2004) e auxílio de microscópio estereoscópico no Laboratório de Hymenoptera, do Instituto Nacional de Pesquisas da Amazônia.

\section{RESULTADOS E DISCUSSÃO}

Foram obtidos 384 espécimes pertencentes a duas tribos (Epiponini e Polistini), 12 gêneros e 38 espécies de vespas sociais (Tabela 1). Polybia foi o gênero mais representativo, com o maior número de espécies (14), seguido de Agelaia (seis espécies) e Apoica (quatro espécies). Os gêneros Asteloeca, Brachygastra, Epipona, Parachartergus e Pseudopolybia foram os menos representativos com uma única espécie.

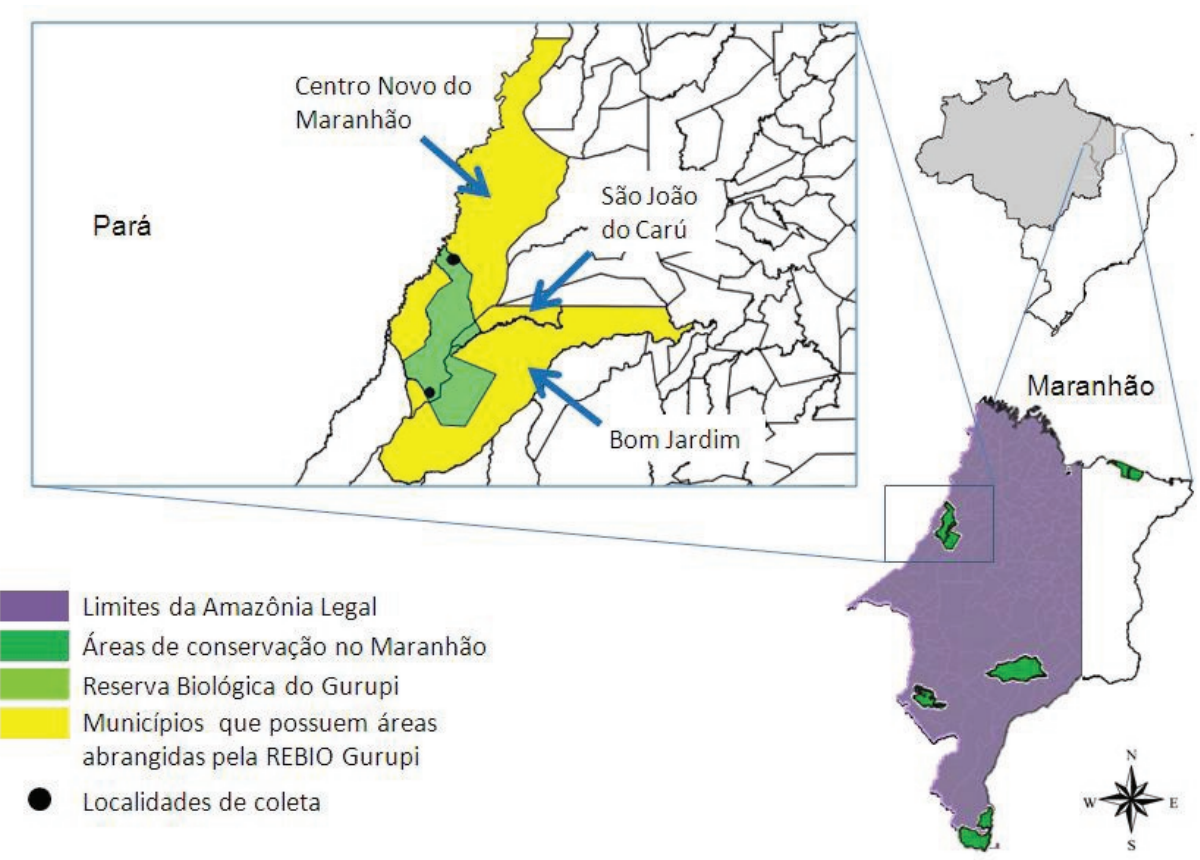

Figura 1. Mapa do Brasil e do estado do Maranhão com as áreas de conservação, em destaque a Reserva Biológica do Gurupi (REBIO Gurupi), no noroeste do estado. 
Tabela 1: Espécies de vespas sociais coletadas na Reserva Biológica do Gurupi, Amazônia Maranhense, com o respectivo método de coleta, os meses em que as vespas foram coletadas e o número total de indivíduos de cada espécie.

\begin{tabular}{|c|c|c|c|}
\hline Táxons de Vespas sociais & Método de Coleta & Meses & $\begin{array}{c}\mathrm{N}^{\circ} \mathrm{de} \\
\text { Espécimes }\end{array}$ \\
\hline \multicolumn{4}{|l|}{ Epiponini } \\
\hline Agelaia angulata (Fabricius) & Malaise & julho & 4 \\
\hline Agelaia centralis (Cameron)* & Malaise, Suspensa, Luminosa & janeiro, maio, novembro & 10 \\
\hline Agelaia fulvofasciata (DeGeer) & Colônia, Malaise, Suspensa, Luminosa, McPhail & $\begin{array}{l}\text { janeiro, março, setembro, } \\
\text { novembro }\end{array}$ & 70 \\
\hline Agelaia myrmecophila (Ducke)* & Luminosa & janeiro & 3 \\
\hline Agelaia pallipes (Olivier) & Colônia, Malaise, McPhail & março, maio, novembro & 9 \\
\hline Agelaia testacea (Fabricius)* & Colônia, Malaise, Suspensa, Luminosa, McPhail & $\begin{array}{l}\text { janeiro, maio, setembro, } \\
\text { novembro }\end{array}$ & 107 \\
\hline Angiopolybia pallens (Lepeletier) & Colônia, Malaise, McPhail & março, junho, novembro & 5 \\
\hline Angiopolybia paraensis (Spinola)* & Colônia, McPhail & março & 2 \\
\hline Apoica arborea de Saussure & Luminosa & julho & 7 \\
\hline Apoica pallens (Fabricius)* & Luminosa & janeiro, novembro & 3 \\
\hline Apoica pallida (Olivier) & Luminosa & janeiro, maio & 32 \\
\hline Apoica strigata Richards & Luminosa & janeiro & 1 \\
\hline Asteloeca traili (Cameron)* & Luminosa & maio, julho & 5 \\
\hline Brachygastra augusti (de Saussure) & Luminosa & abril & 5 \\
\hline Epipona tatua (Cuvier) & Luminosa & novembro & 1 \\
\hline Parachartergus fraternus (Gribodo) & Malaise & abril & 1 \\
\hline Polybia bistriata (Fabricius)* & Malaise, Luminosa & $\begin{array}{l}\text { janeiro, junho, setembro, } \\
\text { novembro }\end{array}$ & 17 \\
\hline Polybia chrysothorax (Lichtenstein) & Colônia, Malaise, Suspensa, McPhail & março, maio, setembro, novembro & 8 \\
\hline Polybia depressa (Ducke)* & Malaise & janeiro & 1 \\
\hline Polybia fastidiosuscula de Saussure & Suspensa, Luminosa & janeiro, setembro, novembro & 17 \\
\hline Polybia ignobilis (Haliday) & Malaise & março & 1 \\
\hline Polybia jurinei de Saussure & Malaise & maio & 1 \\
\hline Polybia liliacea (Fabricius) & Colônia, Malaise, Suspensa, Luminosa & janeiro, maio, setembro & 25 \\
\hline Polybia micans Ducke & Malaise & março & 1 \\
\hline Polybia occidentalis (Olivier) & Malaise, Suspensa, Luminosa & janeiro, setembro & 12 \\
\hline Polybia rejecta (Fabricius) & Malaise, Luminosa, McPhail & janeiro, maio, junho, & 9 \\
\hline Polybia scrobalis Richards* & Malaise, Luminosa & novembro & 6 \\
\hline Polybia sericea (Olivier) & Colônia, McPhail & março, maio & 2 \\
\hline Polybia singularis Ducke* & Malaise & novembro & 1 \\
\hline Polybia striata (Fabricius) & Malaise, Luminosa & janeiro, novembro & 5 \\
\hline $\begin{array}{l}\text { Protopolybia bituberculata Silveira \& } \\
\text { Carpenter* }\end{array}$ & Malaise, Suspensa & março, novembro & 2 \\
\hline Protopolybia chartergoides Gribodo* & Malaise, Suspensa & março & 2 \\
\hline Protopolybia exigua (de Saussure) & Suspensa & janeiro & 1 \\
\hline Pseudopolybia vespiceps (de Saussure) & Malaise & julho & 1 \\
\hline Synoeca surinama (Linnaeus) & Malaise, Suspensa, Luminosa & janeiro, maio, & 4 \\
\hline Synoeca virginea (Fabricius)* & Suspensa & março & 1 \\
\hline \multicolumn{4}{|l|}{ Polistini } \\
\hline Polistes testaceicolor Bequaert* & Suspensa & janeiro & 1 \\
\hline Polistes versicolor (Olivier) & Colônia & setembro & 1 \\
\hline Total geral & & & 384 \\
\hline
\end{tabular}

* Novos registros de ocorrência para o Estado do Maranhão.

As espécies Agelaia centralis (Cameron), Agelaia mirmecophyla (Ducke), Agelaia testacea (Fabricius), Angiopolybia paraenses (Spinola), Apoica pallens (Fabricius), Asteloeca traili (Cameron), Polybia bistriata (Fabricius), Polybia depressa (Ducke), Polybia scrobalis Richards, Polybia singularis Ducke, Protopolybia bituberculata Silveira \& Carpenter, Protopolybia chartergoides Gribodo, Synoeca virginea (Linnaeus) e Polistes testaceicolor
Bequaert não foram reportadas por Richards (1978) e Silva et al. (2012), sendo portanto consideradas novos registros de ocorrência para o Estado do Maranhão.

Devido à carência de estudos regionais sobre Vespidae, não há informação suficiente para atribuição das condições "endêmica" ou "ameaçada" às espécies de vespas da Reserva Biológica do Gurupi. Entretanto, como uma parte das espécies é registrada 
pela primeira vez no Maranhão, deve-se considerá-las como insuficientemente conhecidas em respeito à sua distribuição geográfica ou à condição de raridade e são merecedoras de atenção especial, além disso, ainda salientamos grandes vazios amostrais de vespas no Brasil, principalmente na Região Amazônica.

A maior parte das espécies de vespas sociais esteve representada com poucos indivíduos com exceção de Agelaia fulvofasciata (DeGeer), Ag. testacea, Apoica pallida (Olivier) e Polybia liliacea (Fabricius), com mais de vinte espécimes coletados (espécies com frequência absoluta maior que 5\%). A maioria dos registros das vespas ocorreu nos meses de novembro de 2010 e janeiro de 2011, com 16 e 14 espécies registradas, respectivamente, no período final da estação seca e início da estação chuvosa na Amazônia Maranhense.

Das 38 espécies de vespas sociais encontradas, algumas são consideradas comuns nos ambientes amazônicos como $\mathrm{Ag}$. fulvofasciata, Ag. pallipes, Ag. testacea, Angiopolybia pallens (Lepeletier), geralmente coletadas em grandes quantidades no sub-bosque de floresta de terra-firme amazônicas, e Polybia rejecta (Fabricius) comum em áreas de clareiras (SilveIRa 2002; Silva \& Silveira 2009; Somavilla 2012).

Um aspecto incomum dos resultados é a inexistência de coleta de Mischocyttarus, o maior gênero de vespas sociais e com o maior número de espécies endêmicas e que costuma estar bem representado em listas de faunas locais (Silveira 2002; Silva \& Silveira 2009), possivelmente por não ser realizado um método de coleta específico para este grupo, como o método direto de busca ativa com rede entomológica; porém este aspecto merece maior investigação em futuros estudos. Por outro lado, As. traili, Epipona tatua (Cuvier), Po. depressa e Pseudopolybia vespiceps (de Saussure) possuem número de registros reduzidos na Amazônia brasileira, com uma distribuição mais restrita para determinadas micro-regiões (SiLveIRA 2002), fato que merece ser mencionado, pois tais espécies foram coletadas na REBIO do Gurupi.

O número de espécies aqui obtido é relativamente baixo comparado com resultados obtidos em outras áreas estudadas na Amazônia. Um maior número de espécies foi registrado na Reserva Nacional de Caxiuanã, no Pará, com dois inventários identificando 72 e 65 espécies, respectivamente (Silveira 2002; Silva \& Silveira 2009) utilizando o método direto de busca ativa com rede entomológica, além de armadilha Malaise. Para o estado do Amazonas, em Mamirauá e em Alvarães foram coletadas 46 e 42 espécies, respectivamente (SILVEIRA et al. 2008) utilizando o método direto de busca ativa com rede entomológica, além de armadilha Malaise. Para a Reserva Ducke, 58 espécies em uma área de apenas $25 \mathrm{Km}^{2}$ utilizando o método direto de busca ativa com rede entomológica (Somavilla 2012). Porém, as 38 espécies coletadas na REBIO do Gurupi excedeu o número de espécies encontradas na Serra do Divisor no Acre com 20 espécies (Morato et al. 2008), utilizando armadilha Malaise. Foi maior que a Região dos Lagos no Amapá com 31 espécies (Silveira et al. 2008) utilizando o método direto de busca ativa com rede entomológica, e armadilha Malaise e das 36 espécies registradas na Ilha de Maracá em Roraima (RAw 1998) utilizando o método direto de busca ativa com rede entomológica, ou seja, estados que além de domínio Amazônico, possuem outros biomas, como Cerrado.

A adição de diferentes métodos de coleta é uma importante ferramenta para a amostragem da riqueza de vespas sociais de uma área, pois em geral as espécies possuem um comportamento de forrageio variado. A maior parte das espécies de Epiponini (23 espécies - 60\%), principalmente do gênero Polybia, somente foram coletadas utilizando-se armadilha Malaise, demonstrando a eficiência deste método para este táxon. Certamente, em inventários rápidos, a utilização de tal armadilha pode ser uma boa forma de amostrar este grupo de vespas, principalmente

as que tendem a forragear no sub-bosque da floresta. Porém, o uso de armadilha Malaise como única forma de amostragem para vespas sociais pode subestimar a real riqueza de espécies, principalmente dos gêneros Polistes e Mischocyttarus em uma determinada área (SILveIRA 2002), podendo ser um dos fatores que explica a coleta de apenas duas espécies do primeiro e nenhuma do segundo gênero citado, que são mais coletados através de uma busca ativa direcionada.

Apoica concentra seu forrageio no período noturno (Hunt et al. 1995), diminuindo a possibilidade de ser capturada durante o dia por busca ativa. No presente estudo, Apoica arborea de Saussure, Ap. pallens, Ap. pallida e Apoica striata Richards foram capturadas apenas nas armadilhas luminosas, corroborando tal informação. Além destas, outras 15 espécies (totalizando 50\%) também foram atraídas por armadilha luminosa e capturadas durante a utilização deste tipo de armadilha, o que pode confirmar a eficiência desta na captura de vespas sociais de outros gêneros também, desde que tenham colônias próximas à instalação da armadilha.

Dez espécies (26\%) foram coletadas utilizando-se armadilhas suspensas, corroborando que para a Amazônia, onde o dossel da floresta é elevado, esta técnica é uma das mais eficientes para a coleta de vespas sociais. Ainda, para oito espécies (21\%) pertencentes a Agelaia, Angiopolybia e Polybia a armadilha atrativa, utilizando-se como isca suco de laranja, foi eficiente, uma vez que tais táxons são altamente atraídos por substâncias com alto teor glicídico. Porém, a utilização apenas de armadilhas atrativas como única forma de amostragem para vespas sociais pode subestimar a real riqueza de espécies de Polistes e Mischocyttarus e até mesmo de Epiponini em uma determinada área, devendo ser usada de forma complementar outras técnicas de coleta mais direcionadas para Vespidae. Por exemplo, no trabalho de Clemente (2009) das 21 espécies coletadas em área de floresta em Minas Gerais, 18 foram capturadas na busca ativa, entre as mais frequentes espécies de Polistes e Mischocyttarus e apenas sete espécies em outro tipo de armadilha atrativa, principalmente de Agelaia e Polybia.

Este é o primeiro trabalho para a Amazônia Maranhense registrando 38 espécies nesta reserva, destas, 14 espécies são novos registros para o estado do Maranhão, revelando a importância desta região para a conservação destes insetos. Além disso, o número de espécies atualmente registradas para o estado do Maranhão aumenta de 58 para 72 espécies.

Alguns fatores merecem destaque, sendo um deles a averiguação mais minuciosa nesta área, devido principalmente, ao fato do gênero Mischocyttarus ser o mais especioso entre as vespas sociais e não ser constatada a ocorrência do mesmo neste trabalho. Possivelmente por não ser realizado um método de coleta específico para este grupo, como o método direto de busca ativa como a busca ativa com redes entomológicas de vespas em pleno voo ou em ato de visitação floral. A ocorrência de As. traili, Ep. tatua, Po. depressa, Ps. vespiceps, dificilmente coletadas em inventários na Região Amazônica podem expressar a importância desta área preservada na manutenção das vespas sociais.

\section{AGRADECIMENTOS}

Agradecemos ao Dr. Francisco Limeira de Oliveira pelo convite em determinar as vespas sociais coletadas na REBIO Gurupi. Aos revisores pelas excelentes contribuições ao manuscrito. Ao Programa de Pesquisa em Biodiversidade (PPBio) e ao CNPq/ MCTI - Amazônia Oriental (Processo 558287/2009-3) pelo financiamento da pesquisa e coletas, ao ICMBio, pela autorização de pesquisa na REBIO Gurupi. O primeiro autor agradece o projeto de Pesquisa em Unidades de Conservação do Bioma Caatinga, CNPq/ICMBio 13/2011, pela possibilidade de conhecer a Coleção Zoológica do Maranhão. 


\section{REFERÊNCIAS}

Carpenter, J.M., 2004. Synonymy of the genus Marimbonda Richards, 1978, with Leipomeles Mobius, 1856 (Hymenoptera: Vespidae: Polistinae), and a new key to the genera of paper wasps of the new world. Americam Museum Novitates, 3456: $1-16$.

Carpenter, J.M. \& O.M. Marques, 2001. Contribuição ao Estudo dos Vespídeos do Brasil. Universidade Federal da Bahia, Departamento de Fitotecnia. Série Publicações Digitais, v. 3 , CD-ROM. 147p.

Clemente, M.A., 2006. Vespas sociais (Hymenoptera, Vespidae) amostradas no Parque Estadual do Ibitipoca-MG: estrutura, composição e visitação floral. (Dissertação, Mestrado) Programa de Pós-Graduação em Ciências Biológicas/ Universidade Federal de Juiz de Fora. 79p.

Gressitt J.L. \& M.K. Gressitt, 1962. An improved Malaise trap. Pacific Insects, 4: 87-90.

Hermes, M.G. \& A. Köhler, 2006. The flower-visiting social wasps (Hymenoptera, Vespidae, Polistinae) in two areas of Rio Grande do Sul state, southern Brazil. Revista Brasileira de Entomologia, 50: 268-274.

Hunt, J.H.; R.L. Jeanne \& M.B. Keeping, 1995. Observations on Apoica pallens, a nocturnal Neotropical social wasp (Hymenoptera: Vespidae, Polistinae, Epiponini). Insectes Sociaux, 42: 223-236.

IBAMA, Instituto Brasileiro do Meio Ambiente e dos Recursos Naturais Renováveis. 1999. Plano de Manejo da Reserva Biológica do Gurupi. Brasília. 285p.

La Salle, J., 1993.Parasitic Hymenoptera, biological control and biodiversity. p. 197-215. In: La Salle, J. (Org). Hymenoptera and Biodiversity. CAB. International, Wallingford. 348p.

Morato, E.F., S.T. Amarante \& O.T. Silveira, 2008. Avaliação ecológica rápida da fauna de vespas (Hymenoptera, Aculeata) do Parque Nacional da Serra do Divisor, Acre, Brasil. Acta Amazonica, 38: 789-798.

Rafael, J.A. \& I.S. Gorayeb, 1982. Uma nova armadilha suspensa e primeiros registros de mutucas de copas de árvores. Acta Amazonica, 12: 232-235.

Raposo Filho, J.R. \& V.M. Rodrigues, 1983a. Comportamentos tróficos de Mischocyttarus (Monocyttarus) extinctus Zikán, 1935 (Polistini, Vespinae). I. Alimentação Protéica. Naturalia, 8: 101-104.

Raposo Filho, J.R. \& V.M. Rodrigues, 1983b. Comportamentos tróficos de Mischocyttarus (Monocyttarus) extinctus Zikán, 1935 (Polistini, Vespinae). II. Alimentação Glucídica. Naturalia, 8: 105-107.
Raw, A., 1998. Social Wasps (Hymenoptera, Vespidae) of the Ilha de Maracá. p. 311-325. In: Ratter, J.A. \& W. Milliken (Eds). Maracá. Biodiversity and environment of an Amazonian Rainforest. Chichester, John \& Sons, 508p.

Resende, J.J., G.M.M. Santos, C.C. Bichara Filho \& M. Gimenes, 2001. Atividade diária de busca de recursos pela vespa social Polybia occidentalis occidentalis (Olivier, 1791) (Hymenoptera, Vespidae). Revista Brasileira de Zoociências, 3: 105-115.

Richards, O.W., 1978. The social wasps of the Americas (excluding the Vespinae). London: British Museum of Natural History, 580 .

Silva, S.S., G.G. Azevedo \& O.T. Silveira, 2012. Social wasps of two Cerrado localities in the northeast of Maranhão state, Brazil (Hymenoptera, Vespidae, Polistinae). Revista Brasileira de Entomologia, 55: 597-602.

Silva, S.S. \& O.T. Silveira, 2009. Vespas sociais (Hymenoptera, Vespidae, Polistinae) de floresta pluvial Amazônica de terra firme em Caxiuanã, Melgaço, Pará. Iheringia, Série Zoologia, 99: 317-323.

Silveira, O.T., 2002. Surveying Neotropical Social Wasps. an Evaluation of Methods in the "Ferreira Penna" Research Station (ECFPn), in Caxiuanã, PA, Brazil (Hymenoptera, Vespidae, Polistinae). Papéis Avulsos de Zoologia, 42: 299323.

Silveira, O.T., S.V. da Costa Neto \& O.F.M. da Silveira, 2008. Social wasps of two wetland ecosystems in Brazilian Amazonia (Hymenoptera, Vespidae, Polistinae). Acta Amazonica, 38: $333-344$.

Somavilla, A., 2012. Aspectos gerais da fauna de vespas (Hymenoptera: Vespidae) da Amazônia Central, com ênfase na Reserva Ducke, Manaus, Amazonas, Brasil. (Dissertação Mestrado) Programa de Pós-Graduação em Entomologia/ Instituto Nacional de Pesquisas da Amazônia. 198p.

Somavilla, A. \& A. Köhler, 2012. Preferência floral de vespas (Hymenoptera: Vespidae) no Rio Grande do Sul, Brasil. Entomobrasilis, 5: 21-28.

Sühs, R.B., A. Somavilla, A. Köhler \& J. Putzke, 2009. Vespídeos (Hymenoptera, Vespidae) vetores de pólen de Schinus terebinthifolius Raddi (Anacardiaceae), Santa Cruz do Sul, RS, Brasil. Revista Brasileira de Biociências, 7: 138-143.

\section{Recebido em: 05/12/2013}

Aceito em: 21/05/2014

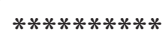

\section{Como citar este artigo:}

Somavilla, A., D.W.A. Marques, E.A.S. Barbosa, J.S. Pinto Junior \& M.L. Oliveira, 2014. Vespas Sociais (Vespidae: Polistinae) de uma Área de Floresta Ombrófila Densa Amazônica no Estado do Maranhão, Brasil, 7 (3): 183-187.

Acessível em: doi:10.12741/ebrasilis.v7i3.404 